Renée A. Walsh

11 décembre 2008

Professeur Durham, Théorie littéraire

Composition finale

\title{
Vers les « lignes de fuite » : une écriture à venir dans la littérature mineure francophone
}

Dans leur livre au sujet de Franz Kafka, Gilles Deleuze et Félix Guattari ont écrit un chapitre intitulé, «Qu'est-ce qu'une littérature mineure ? » où ils se proposent de définir ce qui qualifie la littérature mineure produite dans une langue majeure. Selon Deleuze et Guattari, cette littérature mineure s'articulent autour de trois principes clés, dont les suivants : « la déterritorialisation de la langue, le branchement de l'individuel sur l'immédiat-politique, l'agencement collectif d'énonciation » (33). Dans cette dissertation, nous allons nous adresser à ces trois caractérisations de la littérature mineure à travers trois œuvres de fiction : Nedjma de Kateb Yacine, Prochain épisode d'Hubert Aquin et Les rêveries de la femme sauvage d'Hélène Cixous. En outre, le but de cette dissertation n'est pas de discourir longuement sur chacun des œuvres, mais plutôt d'examiner leur inscription dans ce que Deleuze et Guattari nomment une « littérature mineure ». Nous allons également ponctuer notre analyse avec quelques références à Jacques Derrida dans son essai intitulé, Le Monolinguisme de l'autre. Nous verrons que ces trois œuvres en tant que représentatives d'une littérature mineure, emploient des langages très particulières à leurs projets. En dernier lieu, nous allons établir une mise en comparaison de ces trois œuvres à travers leurs figures de mobilité, autrement dit les transversales qui parcourent les textes (Deleuze, 153). Il s'avère que pour ces trois écrivains, la nation n'est pas représentable_elle est plutôt une promesse à venir. Enfin 
l'écriture de ces trois écrivains se distingue par la relation qu'ils entretiennent avec la nation ou territoire de leur naissance.

\section{Déterritorialisation de la langue}

Tous les écrivains dont nous discuterons dans cette dissertation tombent sous les auspices d'une production littéraire mineure. Néanmoins, ils se distinguent par leurs liens à la langue française. Juifs sépharades grandissant en Algérie-française, Jacques Derrida et Hélène Cixous articulent leurs pensées en français dû au hasard d'une colonisation de ce pays. Kateb Yacine choisit de commencer sa carrière d'écrivain avec la langue française avec les romans de Nedjma et Le Polygone étoilé, avant de se consacrer définitivement à un théâtre populaire en arabe. Hubert Aquin fut un révolutionnaire et écrivain québécois qui étendit la question de la libération du Québec aux autres révolutions anti-coloniales. Aquin s'engagera dans le Rassemblement pour l'indépendance nationale (RIN) et il fut arrêté pour la possession des armes à feu avant de devenir brièvement interné dans un hôpital psychiatrique (Weinnmann, 184). Aquin s'est suicidé en 1977.

D'abord chez Deleuze et Guattari, la question d'une littérature mineure repose sur la géographie et le rapport d'une langue à l'État; elle peut également se jouer entre la tension existant entre la langue vernaculaire, langue parlée à la maison et la langue véhiculaire, langue de mobilité (46). Deleuze et Guattari établissent leur explication d'une littérature mineure à travers l'œuvre de Kafka, juif habitant à Prague qui a cependant écrit en allemand. À partir de là, le décalage géographique entre la langue et le territoire d'un écrivain a amené Deleuze et Guattari à poser la question suivante : «Combien de gens aujourd'hui vivent dans une langue qui n'est pas la leur ? » (35). Or, 
que veut dire l'appartenance à une langue ? Selon Derrida, une langue n'appartient à personne, même s'il demeure possible de se la réapproprier :

La libération, l'émancipation, la révolution, ce sera nécessairement le second tour. Il affranchira du premier en confirmant un héritage en l'intériorisant, en se le réappropriant - mais seulement jusqu'à un certain point, car c'est mon hypothèse, il n'y a jamais d'appropriation absolue. Parce qu'il n'y a pas de propriété naturelle de la langue...(46).

Autrement dit, il n'y a pas primordialement de propriété de la langue. Cependant, il demeure possible de se réapproprier la langue en la déformant, en la greffant afin d'établir, « son propre patois, son tiers monde à soi, son désert à soi » (D\&G, 33). En outre, Derrida précise que la langue s'établit à travers deux figures d'autorité-le maitre de l'école et la métropole— «la Ville-Capitale-Mère-Patrie » (73). La Métropole est modèle «du bien-parler et du bien écrire » (73). D'autant plus, pour le sujet habitant hors de la métropole, elle incarne l'étrangeté puisque représentative d'un Ailleurs.

En tant que juif sépharade de l'Algérie, Jacques Derrida illustre merveilleusement les enjeux de la littérature mineure. En outre, Derrida parle longuement des problématiques des juifs sépharades et ashkénazes avec la langue de l'hôte à travers le XXe siècle (91). En citant Kafka et Paul Celan, Derrida souligne que pour ces écrivains, « l'allemand ne fut néanmoins ni une langue d'adoption ou d'élection (la chose fut, on le sait, plus compliquée) ni, à la différence du français pour les Juifs d'Algérie, une langue "coloniale" ou une "langue du maître" » (112). En dépit des différences qui séparent chacun de ces écrivains de leur rapport unique avec la langue de l'écriture, il n'en reste pas moins que tous ces écrivains entretiennent une distance avec cette même langue, autrement caractérisée par celle d'une production littéraire mineure. 
Deleuze et Guattari ne s'adressent pas à ce qui incite la parole de l'écrivain sauf qu'ils suggèrent que l'écriture se motive par une expression révolutionnaire, d'un « cri » de l'écriture : «Se servir de la syntaxe pour crier, donner au cri une syntaxe. Il n'y a de grand, et de révolutionnaire, que le mineur » (48). Par exemple, l'idée d'un « cri » de l'écriture, d'une abolition totale à travers l'expression est un thème qui apparaît souvent chez Assia Djebar, notamment dans Les Nuits de Strasbourg. Dans les dernières lignes de ce roman, la protagoniste, Thelja, monte la tour de la cathédrale de Strasbourg pour se lancer dans le vide : «Je ne redescendrai pas : après la nuit et juste avant le jour, le vide règne là-bas, debout, un cri dans le bleu immergé... " (Djebar, 405). Le vide, c'est le sublime, c'est anéantissement total à travers l'écriture.

En outre, Deleuze et Guattari parlent du décalage entre l'énonciation et l'écriture : « Disjonction entre contenu et expression. Parler, et surtout écrire, c'est jeûner » (D\&G, 36). Si l'écriture implique le jeûne, elle suggère un genre d'appauvrissement ou de privation. Dans un sens littéral, ceci peut expliquer le style épuré d'un écrivain tel que chez Samuel Beckett. Or, que dire du style katebien de l'écriture, véritable style baroque, fondé sur l'amoncellement des significations à l'infini ? Nous pouvons suggérer que ces deux styles-l'épuré et le baroque réposent sur une exagération, une hyperbole et son contraire, une sous-éxagération, soit une poétique de minimalisme. Les deux styles constituent des exemples du langage unique de l'écrivain d'une littérature mineure.

L'écriture que explique-t-elle de la distance qui sépare la métropole de l'écrivain d'une littérature mineure ? Il me semble, qu'à l'origine, cette langue devient l'expression d'un certain deuil pour l'irreprésentable, autrement dit l'innommable. Selon Derrida, cette énonciation, «la formation du dire-je, du moi-je, ou l'apparition, comme telle, 
d'une ipséité pré-égologique...se serait alors formé, ce je, dans le site d'une situation introuvable, renvoyant toujours ailleurs, à autre chose, à l'autre en général » (55). Ainsi le « uncanny, unheimlich» représentent « une langue innombrable $»($ Derrida, 55). En dernier lieu, Derrida exprime son deuil devant le fait de ne pas posséder d'autre langue maternelle que le français, qui n'est pas sa langue maternelle d'ailleurs : «Je n'avais pas de langue pour le grief, ce mot que j'aime à entendre maintenant en anglais où il signifie davantage la plainte sans accusation, la souffrance et le deuil » (60). Derrida distingue entre la perte et le deuil ; selon lui, il n'a jamais eu de langue à perdre (60). Il ressent seulement le deuil du langage qu'il articule, dans ce cas le français.

\section{Histoires inachevées, histoires à venir}

Face au deuil de l'innombrable, l'écrivain se lance dans l'aventure de l'écriture. Selon le langage derridien, l'écrivain «se renvoie ailleurs» vers ce que Deleuze et Guattari nomment les «lignes de fuite créatrices » (49). En faisant ceci, le langage s'approche d'une promesse à venir, idée ressemblant à ce que Derrida nomme le «messianique » (125). Deleuze et Guattari s'approche de cette idée messianique quand ils énoncent que, «la machine littéraire prend ainsi le relais d'une machine révolutionnaire à venir... » $(\mathrm{D} \& \mathrm{G}, 32)$. En dernier lieu, le messianique semble suggérer une certaine mobilité du texte qui se renvoie à l'avenir. Les trois romans ici discuté se distinguent par une narration circulaire qui suggère le messianique. La première ligne de l'œuvre se renvoie à la dernière ligne. Par exemple, dans Nedjma le récit s'ouvre avec la ligne «Lakhdar s'est échappé de sa cellule » qui revient à l'avant-dernière page du roman. Nous retombons sur la même scène dans la prison, seulement à partir d'une 
narration un peu différente. D'ailleurs, les dernières lignes suggèrent que le roman continue puisque les personnages partent sur la route :

--Je vais à Constantine, dit Rachid.

--Allons, dit Lakhdar. Je t'accompagne jusqu'à Bône. Et toi, Mustapha ?

--Je prends un autre chemin.

Les deux ombres se dissipent sur la route (245).

En tant que dernière image du roman, le chemin ou la route que les personnages empruntent suggère une histoire qui continue à se déferler. De même, Hélène Cixous ouvre et clôt son récit avec les mêmes lignes : «Tout le temps où je vivais en Algérie je rêvais d'arriver un jour en Algérie, j'aurais fait n'importe quoi pour y arriver» (9). La dernière ligne reprend la même formulation, "Tout le temps où je vivais en Algérie je rêvais d'arriver un jour en Algérie » (168). Enfin, chez Aquin le livre ouvre avec la suggestion que le narrateur écrira un roman policier où il sera à la poursuite de son adversaire. Or, à la fin du roman, il n'est pas toujours parvenu à tuer son adversaire, autrement dit, son double, appelé H. de Heutz. Le narrateur est toujours à la poursuite de ce même adversaire, alors il se renseigne à imaginer la fin du roman en nous le décrivant: «Je l'abattrai avant même qu'il atteigne le téléphone...Voilà comment j'arriverai à ma conclusion. Oui, je sortirai vainqueur de mon intrigue, tuant $\mathrm{H}$. de Heutz avec placidité pour me précipiter vers toi, mon amour, et clore mon récit par une apothéose » (Aquin, 229). En outre, le narrateur du récit explique qu'il ne terminera pas l'écriture de son roman policier : «Mon récit est interrompu, parce que je ne connais pas le premier mot du prochain épisode » (Aquin, 226). Que dire de ces récits inachevés ? Il me semble que l'ouverture de ces romans suggère précisément l'impossibilité de saisir la nation ou le territoire en son essence. 


\section{« Nedjma ou le poème ou le couteau »}

En 1948, huit ans avant la parution du roman, Nedjma, Kateb Yacine publie le poème intitulé, «Nedjma ou le poème ou le couteau ». Le titre de ce poème porte la fruition de ce qui serait « une œuvre en gestation continuelle » (Graebner, 141). Ainsi, Nedjma, figure allégorique de la nation algérienne, d'un pays, « pas encore venu au monde » devient emblème de la nation algérienne précisément parce que cette nation n’est pas représentable (Yacine, 173). Signifiant « étoile » en arabe, Nedjma incarne un pays qui a subi des vagues de conquérants depuis le temps immémorial. Plus signifiant encore peut-être est la signification du poème qui précède le roman dont le titre suggère que l'acte de l'écriture est analogique à l'inscription du couteau.

À travers le roman de Nedjma, le peuple algérien n'est pas représentable car il est composé d'un sang-mêlé, d'une lignée de polyandrie qui en fait que personne n'est sûre de leurs origines : «car ce pays n'est pas encore venu au monde : trop de pères pour naître au grand jour, trop d'ambitieuses races déçues, mêlées, confondues, contraintes de ramper dans les ruines... »(Yacine, 173). En outre, les personnages du roman sont constamment en train de se déplacer, comme Nedjma elle-même : «De Constantine à Bône, de Bône à Constantine voyage une femme »(172). Nedjma représente, « une femme perpetuellement en fuite... Nedjma est la forme sensible, l'épine, le noyau, mais non pas l'âme, non pas l'unité vivante où je pourrais me confondre sans crainte de dissolution...» (236). Ainsi, Nedjma incarne une figure d'abolition totale- «à l'embouchure du fleuve passionnel », elle devient une «sirène chargée de noyer tous ses prétendants...» (176). Jamais tenable, Nedjma mène ses amants aspirants seulement vers la « dissolution » ou l'abolition. 


\section{Prochain épisode et l'individu politisé}

Publié en 1965, en plein milieu de la Révolution tranquille de Québec, Prochain épisode, comme son titre l'indique, est symbolique d'une révolution à venir. D'ailleurs, le roman se construit sur une temporalité et une géographie brouillé qui le lie aux autres révolutions : «Depuis hier, quelque part entre H. de Heutz et Toussaint Louverture, j'immerge dans l'eau séculaire des révolutions » (Aquin, 127). La première phrase du roman établit une opposition entre la Suisse et le Cuba : «Cuba coule en flammes au milieu du lac Léman pendant que je descends au fond des choses » (Aquin, 9). Cuba qui possède la même forme géographique renversée du lac Léman se met en opposition avec la Suisse. Ainsi, nous commençons un genre de descente aux enfers à la manière d'un Orphée auquel notre narrateur fera référence en citant la chanson « Desafinado » du film Orfeu negro de Marcel Camus (Aquin 24, 40). D'abord l'action a lieu en Suisse, territoire francophone neutre. Or, le récit développera un axe qui oppose Lausanne, Suisse avec Montréal où Aquin fût interné dans un hôpital psychiatrique, lieu auquel le texte fait référence.

Le deuxième chapitre du roman commence avec une phrase qui introduit le brouillage du temps du roman et qui reviendra au fil du texte : «Entre le 26 juillet 1960 et le 4 août 1792, à mi-chemin entre deux libérations et tandis que je m'introduis, enrobé d'alliage léger, dans un roman qui s'écrit à Lausanne, je cherche avidement un homme qui est sorti du Lausanne Palace...»(23). L'année 1960 inaugure le début de la Révolution Tranquille au Québec avec l'élection de Jean Lesage le 22 juin 1960. Selon l'édition critique de Prochain épisode, le 4 août 1792 représente un, « erreur ou fusion des significations du 4 août 1789 (abolition de tous les privilèges de l'Ancien régime) et 
du 9 août 1792 (la fameuse nuit de l'incarcération de Louis XVI et l'abolition des derniers droits féodaux) ?» (Edition BQ, 179 ). Ainsi, Aquin introduit ce brouillage des dates et des lieux révolutionnaires.

Véritable roman baroque, Prochain épisode se présente comme un genre de roman policier où le «je » narrateur se lance dans la poursuite de son adversaire, nommé H. de Heutz. Cependant, le «je» narrateur se brouille avec le « je» de Hubert Aquin, détenu dans un hôpital psychiatrique du Québec. Si écrire et comme l'inscription d'un couteau chez Yacine alors écrire devient synonyme de tirer chez Aquin : «Les pages s'écrivront d'elles-mêmes à la mitraillette : les mots siffleront au-dessus de nos têtes, les phrases fracasseront dans l'air.. » (228). Ainsi, Aquin écrit un «roman futur...déjà en orbite » où l'écriture devient un processus violent : «Je farcis la page de hachis mental, j'en mets à faire craquer la syntaxe, je mitraille le papier nu...» (18). Ce geste violent nous rappelle encore une fois les mots de Deleuze et Guattari qui parle du fait de «se servir de la syntaxe pour crier » (48). D'ailleurs, Prochain épisode s'oriente toujours vers l'avenir, dans une poursuite étourdie qui poursuit la «ligne de fuite» déterritorialisante dont Deleuze et Guattari nous a parlé :

Ce livre est le geste inlassablement recommencé d'un patriote qui attend, dans le vide intemporel, l'occasion de reprendre les armes. De plus, il épouse la forme même de mon avenir...Il s'est tourné globalement vers une conclusion qu'il ne contiendra pas puisqu'elle suivra, hors texte, le point final que j'apposerai au bas de la dernière page...Je rêve plutôt d'un art totalitaire, en genèse continuelle...(122-3)

Le narrateur de l'histoire nous témoigne de l'histoire qu'il est en train d'écrire tout en soulignant que ce livre ne se terminera pas . Le mouvement du texte, la poursuite de l'adversaire en Suisse, la conduite en vitesse d' une voiture à travers un paysage à la fois helvète et québécois nous lance dans la circulation d'une écriture qui ne se terminera pas. 
Dans le roman, le héros à la poursuite de H. de Heutz reçoit un cryptogramme qu'il ne sera pas capable à déchiffrer :

«CINBEUPERFLEUDIARUNCOBESCUBEREBESCUAZURARANOCTIVAGUS » (Aquin, 26).

Selon les recherches des critiques, Aquin a relevé la première partie de ce cryptogramme d'une tablette d'envoûtement qui se trouve au Louvre (Goudreau, 13). Cette tablette montre l'image d'un démon à bord une barque, à la manière d'un Charon, nocher des enfers.Aquin a ajouté le mot-valise de «noctivagus » qui semble être un amalgame de «nocturne » et «naviguer». En outre, l'incapacité du narrateur à déchiffrer le cryptogramme se pose en modèle de son incapacité à nommer le monde :

Je n'arrive pas à réinventer le code de ce message ; et faute de le traduire dans mon langage, j'écris dans l'espoir insensé qu'à force de paraphraser l'innommable, je finirai pas le nommer. Pourtant, j'ai beau couvrir de mots cet hiéroglyphe, il m'échappe et je demeure sur l'autre rive, dans l'imprécision et le souhait (27).

Le narrateur de lance dans son «noyade écrite » à la manière d'une Orphée descendant aux Enfers. «L'autre rive » ici noté serait peut-être celle du fleuve Achéron ou du Styx.

\section{Écriture aimante et le symbole de $\mathrm{K}$.}

Chez Aquin, écrire, c'est comme faire l'amour ou bien comme tirer un pistolet. Ainsi, Prochain épisode devient également une histoire d'amour écrite à une femme blonde, appelée K : «Écrire est un grand amour. Écrire, c'était t’écrire... » (89). Il s'avère que « $\mathrm{K}$ » femme dont le narrateur a perdu mais qu'il aime toujours incarne le Québec. Néanmoins, « K » comme le pays que le narrateur imagine, l'évade : «Où est-il le pays qui te ressemble, mon vrai pays natal et secret, celui où je veux t'aimer et mourir ? (102). Cette phrase est notable pour sa citation du vers de «L'Invitation au Voyage » de Baudelaire : «Aimer à loisir/ aimer et mourir/ Au pays qui te ressemble !» 
(Baudelaire, 84). « $\mathrm{K}$ » représente ce désir d'évasion et le souhait de voir naître un pays idéal dont le narrateur pourrait appeler le sien. Or, l'amour que le narrateur imagine avec « $»$ est un amour de l'abolition de soi : «Ton pays natal m'engendre révolutionnaire : sur ton étendue lyrique, je me couche et je vis. Au fond de ton ventre de nuit, je frappe en m'évanouissant de joie, et je trouve la terre meurtrie et chaude de notre invention nationale » (188).

\section{Le Venant, le messianique et les néologismes chez Cixous}

Récit contemporain, Les rêveries de la femme sauvage n'est pas pour le moindre un texte se voulant « révolutionnaire » comme celui de Aquin ou représentatif d'un pays comme celui de Yacine. Néanmoins, dans ce texte, il s'agit d'une tentative de retrouver des mémoires d'une Algérie où Cixous a grandi et d'une Algérie dont elle était exclue en tant que juive. Au début du texte, L'Algérie reste quelque chose qu'il est impossible à tenir : «...c'est exactement ce qui s'est passé avec Algérie, du temps où j’y vivais : je l'avais, je la tenais-je ne l'avais plus, je ne l'avais jamais eue, je ne l'ai jamais embrassée »(13). Cependant, Cixous introduit des symboles qui représentent la possibilité de tenir, de couver ce pays. L'un de ces symboles est le Vélo, écrit avec un « $\mathrm{v} »$ majuscule, que Jennifer Yee nomme « le messie longtemps attendu »(191). Cixous raconte longuement l'histoire d'un Vélo que Cixous et son frère désirent après la mort de leur père. Quand leur mère achète un vélo de modèle féminin, cet événement devient véritablement castrateur pour son frère. Toutefois, c'est son frère qui s'emparera du vélo pour découvrir les quartiers au-delà de leur appartement. Enfin, ce Vélo porte de multiples significations dans le texte. Il est le «non-venant» pendant les quatre années 
qui séparent leur premier souhait du vélo de son «apparition » tout d'un coup (27). Ce vélo devient un «crime » lorsque son frère doit faire des promenades à bord un vélo féminin (37). Enfin, ce vélo représente à la fois un destin tragique—« ce Vélo fut notre lot », et un désir de connaître le pays de l’Algérie :

Non, ma mère, structurellement, n'était pas faite pour comprendre la gravité de l'affaire du Vélo, elle ne voyait pas, structurellement, que nous étions fous et malades du besoin de l'Algérie, de la réalité intérieure de ce pays qui était notre pays natal et pas du tout nôtre...(57)

À travers le vélo, Cixous et son frère désiraient connaître l'Algérie. Enfin, ce vélo représente la mobilité et ainsi la possibilité de fuite. Cixous met en comparaison le vélo et les livres :

Donc je passais d'un jour à l'autre d'un livre à l'autre sans livres j'aurais sombré, et quels livres n'importe, il suffisait qu'il y ait un volume rectangulaire de la dimension de mon pied sur lequel me poser pour traverser l'abîme, âne, avion, aigle, charrette tout est bon je glisse d'un dos à l'autre...et mon frère cependant fait l'abîme en vélo. Livre et vélo nous transportent l'un comme l'autre...

je lis comme il pédale comme je lis nous pédalisons toutes nos forces emportés par des pentes dangereuses (Cixous, 82-3).

Il est intéressant de noter ici le néologisme «pédalisons » pour connoter le mouvement. En outre, le langage établi par Cixous pour son récit ne suit ni les règles ni la syntaxe normale de la langue française. De préférence, elle écrit comme si le langage coulait d'un seul trait, avec une forte capacité de transcrire le registre oral à l'écrit.

Cixous crée toute une série de néologismes dans son récit pour exprimer ce qui serait autrement inexprimable. Nous pouvons noter l'expression « malgérienne » $(111,125)$ ou encore « inséparabe », un amalgame des mots «inséparable » et «arabe » (Cixous 89, 45). «Inséparabe » sert à refléter la tension ressentie par Cixous qui voulait connaître les arabes de son quartier mais en était empêché par les relations entre les communautés. 
«Malgérienne » sert à refléter son sentiment d'être mal à l'aise dans un pays où elle n'était pas «l'invitée » et où elle ressentait une souffrance-《une algie » (16). En outre, Jacques Derrida souligne la qualité unique du langage de Cixous, en disant qu'en se rendant compte de ses origines diverses, elle, «tresse toutes ces filiations, les réengendrant vers un avenir encore sans nom » afin de, « réinvente[r], entre autres, la langue de son père, sa langue française, une langue française inouïe... » (Derrida, 113-4). Effectivement, Cixous établit sa propre langue et son propre lexique afin exprimer ses souvenirs de l’Algérie.

\section{Exile et la représentation de la nation :}

Pour établir une synthèse, malgré le fait que les trois œuvres ici discutées viennent d'époques et de lieux différents, nous pouvons trouver des points communs. À travers ces trois œuvres, nous pouvons constater de la difficulté de saisir ou de «nommer» la nation. En pensant à son passé, Cixous, qui a grandi dans une Algérie où elle n'était pas l'invitée résume: «je suis hors de ma maison, j'ai la nostalgie de ce qui n'existera jamais » (112). Autrement dit, il n’y a pas de retour possible en Algérie pour Cixous, pas de «nostos ». Or, elle ressent très forte un sentiment d' «algie », de souffrance. De même, le narrateur de Prochain épisode, après avoir perdu son amour nommé « $\mathrm{K}$ », se ressent condamné à l'exil : «Je marche en pays étranger comme un homme qui vient de te perdre après t'avoir retrouvée par hasard et dans la joie, dans une rue de Lausanne et au fond d'un lit romantique à l'Hôtel d'Angleterre...Je n'ai plus de pays, on m'a oublié » (Aquin, 204). Enfin, chez Yacine, le personnage de Rachid n'arrivera jamais à tenir le personnage de Nedjma, emblématique de la nation algérienne. Faute de la saisir, il est condamné à voyager sans suite et à rôder autour des ruines de Carthage et de Cirta : 
«Sous les palmes de Bône m'attendaient d'autres ruines où je devais rampais comme un lézard délogé de son terrier»(Yacine, 166). Les voix narratives de ces œuvres sont «hors de leurs maisons », se tendant vers les « lignes de fuite » déterritorialisantes où ils peuvent établir leurs propres voix dans les interstices.

\section{BIBLIOGRAPHIE :}

AQUIN, Hubert. Prochain épisode. Montréal : Bibliothèque Québécoise, 2007.

AQUIN, Hubert. Prochain épisode. Paris : Editions Robert Laffont, 1966.

BAUDELAIRE, Charles. Les fleurs du mal. Paris : Editions Gallimard, 1996.

CIXOUS, Hélène. Les rêveries de la femme sauvage : scènes primitives. Paris : Galilée, 2000.

DELEUZE, Gilles. Proust et les signes. Paris : PUF, 2003.

DELEUZE, Gilles et GUATTARI, Félix. Kafka : pour une littérature mineure. «Qu'estce qu'une littérature mineure ?» Paris : Les Editions du Minuit, 2001 : 29-50.

DERRIDA, Jacques. Le Monolinguisme de l'autre ou la prothèse d'origine. Paris : Galilée, 1996.

GOURDEAU, Jacqueline. "Prochain épisode : l'incidence autobiographique ». Etudes littéraires. 17.2 (1984) : 311-332. Disponible : http://id.erudit.org/iderudit/500649ar

JAMESON, Fredric. «Euphorias of Substitution : Hubert Aquin and the Political Novel in Québec ». Yale French Studies. 65 (1983) : 214-223.

WEINMANN, Heinz. Littérature québécoise : des origines à nos jours. Montréal : Editions Hurtubise HMH, 1996.

YACINE, Kateb. Nedjma. Paris : Editions du Seuil, 1996.

YEE, Jennifer. "The Colonial Outsider : "Malgérie" in Hélène Cixous's Les rêveries de la femme sauvage ». Tulsa Studies in Women's Literature. 20.2 : (Autumn, 2001) : 189-200. 